\title{
LA CARRERA DEL PARAGUAY A FINES DEL SIGLO XVIII
}

\section{THE CARRERA OF PARAGUAY AT THE END OF THE 18TH CENTURY}

\author{
Isabel Paredes* \\ Universidad Nacional de Tres de Febrero, Buenos Aires, Argentina <isahistoria@yahoo.com.ar>
}

Resumen. Durante las tres últimas décadas del siglo XVIII Paraguay vivió una serie de transformaciones políticas y económicas que han permitido considerar esa época como la edad de oro. Al impulso adquirido con la creación del régimen de Intendencias, se deben agregar los beneficios surgidos de las medidas económicas de 1778. El uso exhaustivo de las Guías y Tornaguías de Aduana permite abrir caminos de investigación a partir de los datos que ellas aportan. En este artículo se hace hincapié en los partícipes del tráfico fluvial a fin de mostrar la diversificación que practicaban y que les permitía tener injerencia en toda la práctica mercantil de la provincia y su conexión con otros centros de intercambio.

Palabras clave: Paraguay; transporte fluvial; transportista; comercio.

Abstract. During the last three decades of the 18th century Paraguay underwent a series of political and economic transformations which have allowed for the consideration of the period as the Golden Age. To the impetus acquired with the creation of the Mayoralties Régime, we must add the benefit derived from the economic measures taken in 1778. The thorough use of the Customs guides and tornaguias permitted to open ways of investigation starting from the data provided by them. On this article, the emphasis is laid on the protagonists intervening in the fluvial trade so as to show the diversification which they performed and which allowed them to hold a strong influence on the whole mercantile practice of the province and its connections with other interchange centers.

Key words: Paraguay; river navigation; transport worker; commerce.

Fecha de recepción: marzo de 2013. Fecha de aceptación: junio de 2013.

\footnotetext{
* Integrante del proyecto de investigación La Circulación Mercantil Interna en el Área Rioplatense a Fines del Siglo XVIII, dirigido por el doctor Fernando A. Jumar, bajo financiamiento de la Universidad Nacional de Tres de Febrero, Argentina.
}

Am. Lat. Hist. Econ., año 21, núm. 1, enero-abril, 2014, pp. 66-91 


\section{INTRODUCCIÓN}

$\mathrm{L}$ os estudios referidos al comercio de Paraguay, subregión integrante del espacio rioplatense, se han centrado especialmente en la presencia jesuítica y en la época emancipadora. Sin embargo, en las últimas décadas se intensificaron las investigaciones que analizan el papel que este centro mercantil desempeñó en el último tercio del siglo XVIII. ${ }^{1}$ Para esa época se conjugó en Paraguay una serie de medidas y circunstancias que provocaron importantes cambios en la participación que la región representó en el espacio rioplatense, cada vez más afianzado. La expulsión de la orden jesuítica provocó cambios administrativos, jurisdiccionales y demográficos; la abolición del puerto preciso en Santa Fe dio mayor maniobrabilidad mercantil; el establecimiento del Real Estanco de Tabacos permitió la afluencia de metálico a la zona, aunque mayoritariamente se siguió usando la moneda de la tierra; la Real Ordenanza de Intendentes le dio a la nueva autoridad un margen de acción y centralización de decisiones que reordenaron la provincia. Simultáneamente, el mayor control en la salida y entrada de productos por el puerto asunceno produjo el asentamiento de las guías y tornaguías del tráfico fluvial, eje primordial del intercambio.

En la propuesta que aquí se presenta se han elegido como objeto de estudio los protagonistas del comercio legal, ya sea en su papel de transportistas, como de comerciantes y de productores o, algo frecuente, en el desempeño múltiple de estas actividades por parte de una misma persona, analizando, también, cuáles eran sus vinculaciones en los distintos puntos de destino. Se considerará que el eje estaba en el dominio del transporte, que permitió el traspaso de poder de los pobladores de familias tradicionales a llegados recientes, ya fuesen peninsulares o americanos procedentes de otras regiones, es por ello que el dueño de las embarcaciones que unían los puertos principales entre Asunción y Buenos Aires es el partícipe fundamental de este panorama.

\section{LAS FUENTES Y SUS LIMITACIONES METODOLÓGICAS}

Para lograr el objetivo propuesto, como fuente de base se utilizarán las guías y tornaguías conservadas en el Archivo Nacional de Asunción (ANA). Estos registros comenzaron a asentarse a partir de 1783 por parte de los funcionarios radicados en la ciudad de Asunción.

\footnotetext{
${ }^{1}$ No se pueden dejar de mencionar los aportes hechos, especialmente en las décadas de 1970 y 1980, por Romero de Viola, Paraguay, 1987; Cooney, Economía, 1990, y Whigham y Cooney, Campo, 2006.
} 
Cada asiento deja constancia del número de registro; fecha; persona que comparece para declarar un envío, si lo hace por cuenta propia, a nombre de otro o como fiador de un tercero; la mercadería que se envía y su cantidad; lugar de salida; destino; medio de transporte y dueño del mismo; receptor de la mercadería en destino; testigos del trámite y, en ocasiones, la procedencia del producto.

La fuente presenta ciertas limitaciones, la más importante es que sólo refleja el flujo legal de mercancías que pasaba por el puerto de Asunción. Queda de lado, entonces, el tráfico directo que escapaba a los controles oficiales y el movimiento interno entre pequeñas poblaciones. Sin embargo, el seguimiento de los productos que tenían por finalidad el comercio en una escala mayor se veía precisado a registrar su salida, y se considera suficiente muestra como para arribar a conclusiones válidas respecto al objeto de estudio. Otra limitación, impuesta por el tiempo y las condiciones de conservación, es el deterioro que presentan las guías de algunos años, que las hace prácticamente ilegibles, y lo que se puede rescatar de ellas sirve para análisis cualitativo parcial, pero no para un análisis cuantitativo.

Antes de comenzar a aportar datos cuantitativos se hace necesaria una aclaración metodológica: se imputarán las cargas al año en el que se las registra, al margen de que el viaje puede haberse iniciado en el año calendario posterior. Si se imputan los datos a los meses de noviembre y diciembre, por ejemplo, es probable que la marcha se haya iniciado en enero o febrero del año siguiente. La fuente indica cuándo se registra, pero no aclara cuándo comienza la travesía, por lo que hubo que elegir un corte temporal en los análisis y se optó por lo indicado, haciendo referencia a las cargas anuales y no a la cantidad de viajes. Se aclara también que, a fin de simplificar la presentación, se han seleccionado los bienios de la década de 1780 para los ejemplos, ya que las conclusiones generales no sufren alteración y, de ser necesario, en los otros años se aclararán casos puntuales. ${ }^{2}$

Los datos obtenidos se han analizado y entrecruzado para establecer la importancia de los transportistas a considerar. A fin de seleccionar un grupo para analizar las cuestiones relacionadas con el transporte de mercaderías por la vía fluvial, se hizo un relevamiento de los datos aportados por las guías y tornaguías y se optó por los grandes transportadores, que en su conjunto representan entre 32 y $50 \%$ de las cargas anuales. ${ }^{3} \mathrm{Si}$ bien no fueron los únicos dueños de embarcaciones de gran porte y relaciones comerciales de importancia, también se tuvo en cuenta a qué otras actividades se dedi-

\footnotetext{
${ }^{2}$ Toda la información de guías y tornaguías en Archivo Nacional de Asunción (en adelante ANA), sección Nueva Encuadernación, en los volúmenes correspondientes a cada año.

${ }^{3}$ Cálculo obtenido con base en datos del cuadro 1 y los totales anuales aportados por Cooney, "Yerbatales", 2006, p. 47.
} 
caban y que su lugar de asiento fuera Paraguay. De esa manera se decidió trabajar con Luis Rodrigo Baldovinos, José González de los Ríos, Juan Machain, Tomás Ortega Fernández, Antonio Sánchez y Juan Zevallos. ${ }^{4}$

\section{APORTES HISTORIOGRÁFICOS}

El objetivo historiográfico de este trabajo es contribuir a completar los estudios ya realizados, en torno a la importancia de la yerba mate, en el trabajo insoslayable de Juan Carlos Garavaglia; al estanco del tabaco, analizado en detalle por Herib Caballero Campos; a la expansión territorial hacia el norte, tema desarrollado ampliamente por Renée Ferrer y posteriormente por Nidia R. Areces, y las modificaciones demográficas y expansivas surgidas a partir de la expulsión jesuítica, tratadas por Ignacio Telesca. ${ }^{5}$

En el caso de la obra de Garavaglia, si bien no llega a tratar el último tercio del siglo XVIII, el minucioso análisis del trabajo y de la comercialización de la yerba mate sigue siendo insustituible, a pesar de los años transcurridos desde su primera publicación. Respecto a la Renta del Tabaco, la investigación de Caballero Campos es amplia y completa, superando lo hecho por otros autores hasta ese momento. En cuanto a Ferrer y Areces, presentan dos perspectivas de una misma cuestión. La obra de la primera se centra en la evolución del núcleo concepcionero, prestando especial atención al asentamiento poblacional y al reparto de tierras en una zona de frontera; aunque su centro de interés es el Paraguay del doctor Francia, Areces permite contextualizar - en la primera parte de su libro- las cuestiones territoriales y comerciales que lograron que, en pocos años, la Villa Real de la Concepción se transformara en el extremo norte de la carrera fluvial. La contribución de Telesca permite explicar los cambios poblacionales que estuvieron ligados a nuevos caminos y circuitos productivos y mercantiles.

Un lugar especial merecen los trabajos pioneros de Jerry Cooney por la minuciosidad en tratar temas que hasta ese momento no habían tenido un análisis específico. ${ }^{6}$ En la misma línea se encuentran las investigaciones iniciales de Thomas Whigham, que integran parte de su libro acerca de $\mathrm{Pa}$ raguay y Corrientes, aunque su objetivo es, primordialmente, proyectar su estudio hacia la etapa independiente a fin de demostrar que el Alto Plata no fue una región estancada. ${ }^{7}$

\footnotetext{
${ }^{4}$ En la grafía de Baldovinos y de Zevallos, acerca de lo cual hay varios registros, se ha optado por utilizar la que ellos mismos usaban para firmar.

${ }_{5}^{5}$ Garavaglia, Mercado, 2008; Caballero, Moneda, 2006; Ferrer, Siglo, 2008; Areces, Estado, 2007, y Telesca, "Expulsos", 2009.

${ }^{6}$ Cooney, Economía, 1990, recopila los trabajos dados a conocer entre 1979 y 1987.

${ }^{7}$ Whigham, Río, 2009, el material inicial de este libro es su tesis doctoral de 1985.
} 
A las obras mencionadas se suman otros trabajos publicados como artículos en revistas especializadas, enfocados en temas puntuales, tal es el caso de Claudia Wentzel, Miguel A. Rosal y Diana Durán. ${ }^{8}$ Si bien los dos primeros fueron publicados hace casi un cuarto de siglo, no han perdido vigencia, aunque serían pasibles algunos ajustes a la luz de nuevas interpretaciones. En el caso de Durán, el análisis de testamentos le permite presentar un panorama general de los españoles peninsulares llegados a Paraguay a fines del siglo XVIII.

En todos los casos apuntados, tanto en las obras de envergadura como en los artículos, se puede observar que abordan una temática específica, con lo que se perdería una visión integral del Paraguay tardocolonial. Es por ello que, a partir de 2008 y utilizando las mencionadas guías y tornaguías de Aduana conservadas en el ANA, se llevó a cabo una serie de estudios que analizaron los problemas que se presentan a la hora de investigar las características del intercambio; el intercambio de productos no tradicionales en la historiografía rioplatense; las características geohistóricas que le dan unidad e integración a la región del Río de la Plata por medio de la red de circuitos y caminos mercantiles, y la participación de un protagonista ineludible en el análisis del comercio hispano colonial, como fue el contrabandista, ${ }^{9}$ a las que ahora se agrega la que aquí se presenta, dedicada a los grandes transportistas.

Para lograr un trabajo ajustado a la realidad de la época es imprescindible, también, tomar distancia de la historiografía tradicional que se ha especializado en marcar fronteras nacionales cuando estas no existían y, dejando de lado el énfasis puesto por dicha corriente para reafirmar un pasado nacional glorioso, se propone pensar a Paraguay como una zona con características económicas locales propias, pero ligada a un todo lo regional que abarcaba el espacio rioplatense. Posicionarse en Paraguay significa que Buenos Aires deja de ser el centro de análisis, a pesar de que la mayor cantidad y valor de productos tuviesen a esta última ciudad como destino final. Desde Asunción se aprecia un abanico de caminos y productos de intercambio imposibles de valorar y evaluar si el foco es la capital virreinal.

\section{LA REGIÓN Y EL TRANSPORTE}

Con la finalidad de clarificar las categorías a utilizar, se considera que se debe iniciar esta explicación con el concepto de espacio y región que se utilizará. Desde el punto de vista de los geógrafos, el espacio es dinámico

\footnotetext{
${ }^{8}$ Wentzel, “Comercio”, 1988; Rosal, “Transporte”, 1990, y Durán, “Inmigrantes”, 2011.

${ }^{9}$ Paredes, "Rutas", 2008; “Comercio”, 2009; "Región”, 2010, e "Ilegalidad”, 2011.
} 
y cambiante, y resulta ser "la 'puesta' en valor y producción del territorio [...] de acuerdo con las determinaciones históricas, económicas, técnicas, culturales, dentro de las cuales se incorporan las variables físicas" . ${ }^{10}$ Es decir, que espacio y región surgen de la relación entre las características territoriales y la acción humana, variable en cada época, circunstancia y necesidad de la sociedad.

En el sentido expuesto, es posible apreciar que el espacio peruano analizado por Carlos Sempat Assadourian ${ }^{11}$ para el siglo XVII y primera parte del siglo XVIII fue perdiendo identidad en la zona rioplatense a medida que esta adquirió vida y sustento propio, a raíz de su crecimiento comercial autónomo con ultramar. A partir de mediados del siglo XVIII se perfila entonces el "espacio rioplatense" que, sin dejar de tener intensa relación con ese espacio peruano, se desprende de él y se resignifica la región.

De tal manera, los territorios aledaños a la cuenca del Plata afianzan sus contactos, crecen en poblaciones y dan lugar a la aparición de nuevos caminos de intercambio. Así, la fundación de pueblos en Entre Ríos, por ejemplo, facilitó el contacto transversal entre la Banda Oriental y Santa Fe, obviando el paso por Buenos Aires. ${ }^{12}$ De manera similar, en Paraguay la disposición demográfica y poblacional de fines del siglo en análisis dio nacimiento a una red dinámica de relaciones sociales y mercantiles que, partiendo de Asunción, se dirigían hacia norte, con la fundación de la Villa Real de la Concepción, y hacia el sur con el establecimiento de Pilar de Neembucú, dando así nuevo fortalecimiento a la carrera Paraguay-Paraná (véase mapa 1).

Condición necesaria para comprender el funcionamiento mercantil que se analizará es conocer las características físicas de los territorios a recorrer. Desde la fundación de Asunción en 1537, la comunicación más directa con el "mundo exterior" fue el eje fluvial que desemboca en el Río de la Plata. Existieron los intentos de comunicación por caminos terrestres hacia el Alto Perú y hacia el Atlántico por Río Grande, que no resultaron convenientes. El del Alto Perú por las dificultades del terreno y, especialmente, por la belicosidad de los indígenas. El de Río Grande porque, a las razones nombradas, se agregó la expansión portuguesa en Brasil.

El eje formado por los ríos Paraguay y Paraná es navegable en toda su extensión, y ello permitió eludir el tránsito terrestre, ya sea en zonas de pueblos originarios hostiles como en terrenos bajos y pantanosos. El siglo XVIII muestra un incesante trajinar de las más variadas embarcaciones tanto de origen europeo (balandras, sumacas), como otras de origen nativo,

${ }^{10}$ Barsky, "Auge", 2001, p. 44.

${ }^{11}$ Assadourian, Sistema, 1983.

${ }^{12}$ Respecto a las nuevas poblaciones en Entre Ríos, véase Djenderedjian, "Economía”, 2003. 


\section{MAPA 1. UBICACIÓN DE LA CARRERA DEL PARAGUAY EN AMÉRICA DEL SUR}

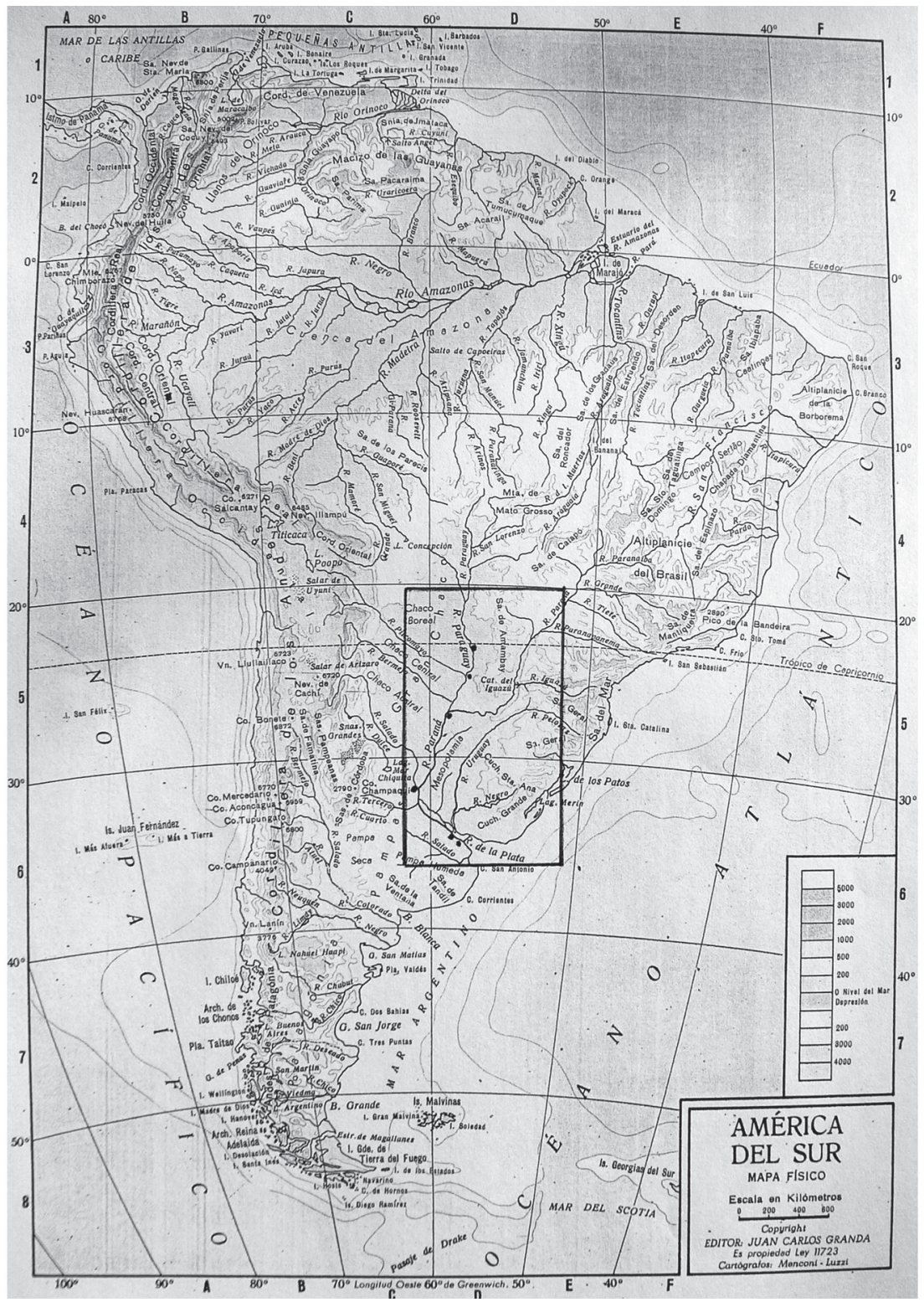

Fuente: elaboración propia con base en Atlas, 1977. 
con las adaptaciones necesarias para asegurar el traslado de cantidades significativas de productos. Por otra parte, los caminos internos fueron, también, en gran medida fluviales. En efecto, los ríos Jejuy y Tebicuary se usaron como rutas ineludibles para llevar los productos hasta el río Paraguay, por lo que el panorama habitual en estos cursos de agua lo constituía la presencia de balsas, canoas, jangadas e itapas. ${ }^{13}$

No obstante lo dicho, el tránsito terrestre por medio de carretas fue necesario cuando los ríos nombrados estaban a considerable distancia de la producción, o fue complementario combinando ambas formas de transporte. Tal es el caso de la comunicación entre Corrientes y las misiones ubicadas entre el Tebicuaty y el Paraná, que en ocasiones se hacía cruzando el Paraná por zonas propicias para pasar las carretas.

La casi imposibilidad de utilizar la ruta terrestre hizo que los ríos $\mathrm{Pa}$ raguay y Paraná fuesen el eje de comunicación. Su descripción fue objeto de estudio desde las primeras apreciaciones de visitantes y funcionarios hispanos en la zona. Siguiendo esas descripciones, se puede decir que ambos ríos resultan navegables, son anchos y con suficiente profundidad para las embarcaciones del siglo XVIII, pero también ofrecen dificultades por las corrientes y la cantidad de islas que se encuentran a lo largo de su curso. ${ }^{14}$ Tal vez una imagen clara de esta vía de agua es la que a principios del siglo xx dejó un escritor catalán: "se ve [...] una isla. Detrás de la isla hay otro curso de agua, y más allá del agua más islas. Más allá de las islas, el río continúa [...] Es un inmenso lago que va bajando [...] una llanura que se desliza."15

Por la intensa utilización de estas vías fluviales, a lo largo del siglo XVIII se formó un rosario de pequeñas poblaciones, embarcaderos y asentamientos en las riberas de ambos ríos que permitía navegar con la seguridad de tener lugar de descanso y aprovisionamiento. Esta presencia de pobladores locales ribereños no respondía originariamente a fundaciones formales, sino que nacieron de la necesidad de cubrir ambos requerimientos. Primero fue el amarradero y luego el pueblo. Las características de "puertos" y "embarcaderos", que no sólo sirvieron para carga y descarga de productos, sino que implicaban una intensa actividad social y laboral en sus cercanías, distan considerablemente de la definición de diccionario de estos términos; en efecto, en origen fueron simples lugares resguardados que permitían asegurar balsas, canoas y garandumbas durante el descanso nocturno. A su alrededor se fueron estableciendo pequeños abas-

\footnotetext{
${ }^{13}$ Las mejores descripciones de los ríos y de las embarcaciones se encuentran en Azara, Diario, 1836; Quiroga, Descripción, 1836, y Alvear, Relación, 2000.

${ }^{14}$ Reitano, "Veleros", 2009, y Wentzel, "Comercio", 1988.

${ }^{15}$ Rusiñol, Barcelona, 1999, p. 199.
} 
tecedores $\mathrm{y}$, fundamentalmente, personas ligadas al comercio directo que introducían a tierra yerba, tabaco y cueros.

\section{EL TRANSPORTE Y SUS RESPONSABLES}

Aquí, como en otras categorías, es necesario hacer un análisis de los diferentes integrantes de esta actividad, para ello se partirá del tipo de embarcaciones que poseían, luego se tendrá en cuenta los lugares de destino y tipo de carga, para, por último, establecer la incidencia que tenían en el tráfico mercantil.

En la categoría de embarcaciones se encontraban, por un lado, las impulsadas básicamente a remo, pértiga o pala, como los botes, las canoas, las balsas simples de dos, tres, cuatro y cinco canoas, las itapá, las piraguas de distinto porte, las garandumbas y lanchas; si bien su propulsión era fundamentalmente dependiente de la fuerza humana, a veces se ayudaban con velas simples. Por otra parte, las embarcaciones de mayor porte y tecnología como sumacas, bergantines y balandras, eran movidas esencialmente a vela, aunque en ocasiones necesitaban de la ayuda de los marineros y peones para impulsar su marcha.

A veces se utilizó diferente nominación para el mismo tipo de transporte y, en otras, la denominación es la misma pero la embarcación tiene diferencias. Ejemplo claro de esto último es el nombrar como bote a la forma de transporte de pequeño porte, impulsado por remos, con poca capacidad de carga y utilizado para trayectos cortos hasta embarcaderos cercanos y llamar así también a los de alijo, que llevaban las embarcaciones mayores para el caso de un eventual naufragio y que supuestamente debían ir vacíos para trasbordar a ellos la carga en peligro, sin embargo, salían de puerto con cargas de más de 1000 arrobas. ${ }^{16}$ Por último, también se llamaba bote a las embarcaciones de mayor tamaño utilizadas por personas dedicadas a la actividad fluvial. Casos ilustrativos son los de Miguel Jerónimo Garmendia y Juan Cuello; el primero de los nombrados llevó en su bote 1028 arrobas, entre yerba, miel, dulces, conservas y azúcar, con destino a Santa Fe; el segundo, a su vez, cargó en el suyo, de Asunción a Corrientes y Santa Fe, productos por 2926 arrobas, llevando yerba, miel, maní, azúcar y algodón. ${ }^{17}$

\footnotetext{
${ }^{16}$ En febrero de 1783, Juan de Zevallos cargó en su bote de alijo 951 arrobas, y entre febrero y marzo del mismo año Juan de Machain cargó el suyo con 1293 arrobas, tal como puede comprobarse en varios asientos registrados en esa época, véase ANA, Nueva Encuadernación, vol. 11, Escrituras, 1783.

${ }^{17}$ La carga de Garmendia se hizo en febrero y la de Cuello entre mayo y junio de 1783. Véase ANA, Nueva Encuadernación, vol. 11, Escrituras, 1783.
} 
También resulta ambigua la identificación bajo la denominación simple de "barco" o "embarcación", lo que no permite, más que por deducción, saber de qué transporte se trata. Generalmente, como barco se supone que se quiere indicar bergantín o sumaca, pero hablar de embarcaciones sin mayor detalle puede aplicarse a cualquier transporte con gran capacidad o poca, a remo o a vela, con quilla o sin ella.

Del grupo elegido para el análisis se puede adelantar que, para la década de 1780, todos estos individuos estaban ya instalados en Paraguay, aunque sus familias no fueran originarias de allí. Los Baldovinos eran de procedencia chilena, González de los Ríos, Machain, Ortega Fernández y Sánchez eran peninsulares, y Zevallos tenía su raigambre familiar en Santa Fe. Todos ellos tuvieron una dotación de embarcaciones de gran porte que utilizaron en viajes separados o en un solo envío en conjunto. Como ejemplo se puede mencionar que Baldovinos, en 1785 y en 1787, realizó dos cargas por año para las provincias de Abajo; en el primer caso lo hizo en marzo llevando yerba en una garandumba y su correspondiente bote, en septiembre lo hizo en un barco y bote; dos años después volvió a viajar en marzo utilizando garandumba, piragua, y bote, y en junio cargó su barco para otra travesía. De igual modo, Juan Machain cargó a comienzos de 1783 barco, bote y piragua, y en julio garandumba y barco. Los ejemplos se repiten en los demás transportistas analizados y en otros que con los años fueron aumentando su participación en la actividad, como Juan Cuello, Sebastián Aramburu y Pedro Nolasco Domeq. Acorde con estos datos es posible afirmar que, si bien la época más propicia para realizar los viajes de Asunción a Buenos Aires era durante el invierno, de junio a agosto, también era posible realizarlos en primavera y verano.

Respecto al destino de los viajes de estos empresarios, el paso por Corrientes era poco frecuente, unos pocos envíos eran a Santa Fe y el grueso tenía como meta Buenos Aires (véase cuadro 1).

Los envíos a los puertos intermedios los realizaban embarcaciones menores, en el caso de Corrientes estaban a cargo, en general, de pequeños transportadores locales que utilizaban canoas o balsas para el traslado. En el caso de Santa Fe hubo quienes, utilizando piraguas y garandumbas, se dedicaron a ese intercambio. Fue común también que comerciantes santafesinos propietarios de sus propias lanchas hicieran el viaje de Santa Fe a Asunción y de regreso llevaran los productos paraguayos; es el caso de Juan Cuello, Francisco Candioti y José Ramón Tarragona. El primero de ellos aparece en 1783 cargando su bote con destino a Santa Fe; en los años posteriores ya registra barco, su trayecto también incluye Buenos Aires y él reside en Asunción, aunque su familia permanece en la ciudad de origen. ${ }^{18}$ En cuanto

\footnotetext{
${ }^{18}$ En 1783 su carga es en poca cantidad para Corrientes y la mayor parte para Santa Fe, en lo que se identifica como "bote". En 1786 ya tiene barcos, y la mayor parte de la carga va a Buenos
} 
CUADRO 1. ENVÍOS A BUENOS AIRES (EN PORCENTAJES)

\begin{tabular}{|c|c|c|c|c|}
\hline Transportista & 1783 & 1785 & 1787 & 1789 \\
\hline Luis Rodrigo Baldovinos & $\begin{array}{l}\text { No hay } \\
\text { envíos }\end{array}$ & 82.75 & 73.91 & 88.23 \\
\hline José González de los Ríos & 100 & $\begin{array}{l}\text { No hay } \\
\text { envíos }\end{array}$ & 100 & 100 \\
\hline Juan Machain & 85.70 & 75 & 90 & 87.5 \\
\hline Tomás Ortega Fernández & $\begin{array}{c}\text { No hay } \\
\text { envíos }\end{array}$ & 72.2 & 100 & $\begin{array}{c}\text { No hay } \\
\text { envíos }\end{array}$ \\
\hline Antonio Sánchez & 100 & $\begin{array}{c}\text { No hay } \\
\text { envíos }\end{array}$ & 80 & 100 \\
\hline Juan Zevallos & 81.57 & 100 & 91.66 & 95.45 \\
\hline
\end{tabular}

Nota: sobre el total de envíos anuales de cada uno.

Fuente: elaboración propia con base en Registro de Guías y Tornaguías de Asunción, en ANA, Nueva Encuadernación.

a Candioti, también en 1783 envió sus propias embarcaciones, que fueron cargadas con una importante cantidad de yerba. En los años siguientes la mercancía le es enviada por otros barcos, entre ellos el de Juan de Zevallos, de quien era familiar. ${ }^{19}$ En el caso de Tarragona, cliente habitual de los abastecedores asuncenos, llegó con sus lanchas en varias ocasiones; por ejemplo, en 1785 retornó con 7603 arrobas de yerba y 370 arrobas de azúcar para diferentes comerciantes y para él mismo. ${ }^{20}$

Otra característica a tener en cuenta es que la yerba representó, casi exclusivamente, el producto que se transportaba (véase cuadro 2). El azúcar aparece en varios registros, pero en cantidades que no tienen proporción con la yerba, y lo mismo sucede con las maderas. Otros productos, como el maní, el algodón, el almidón y algunos pequeños muebles y menaje, se encuentran en mínima cantidad.

Si bien los transportistas elegidos son los que registran mayor número de envíos, las cargas no representan una cantidad medianamente constante, por el contrario, son notorias las diferencias anuales (véase cuadro 3).

Aires. En 1788 envía yerba, azúcar y almidón para su esposa, para gasto de la casa en Santa Fe, en ANA, Nueva Encuadernación, vols. 11, 1 y 150.

${ }^{19}$ Ibid., vol. 11.

${ }^{20}$ Ibid., vol. 79. 


\section{CUADRO 2. ENVÍOS CON YERBA (EN PORCENTAJES)}

\begin{tabular}{|c|c|c|c|c|}
\hline Transportista & 1783 & 1785 & 1787 & 1789 \\
\hline Luis Rodrigo Baldovinos & $\begin{array}{r}\text { No hay } \\
\text { envíos }\end{array}$ & 99.65 & 86.96 & 100 \\
\hline José González de los Ríos & 92.85 & $\begin{array}{l}\text { No hay } \\
\text { envíos }\end{array}$ & 100 & 100 \\
\hline Juan Machain & 96.42 & 75 & 70 & 100 \\
\hline Tomás Ortega Fernández & $\begin{array}{r}\text { No hay } \\
\text { envíos }\end{array}$ & 77.78 & 92.86 & $\begin{array}{r}\text { No hay } \\
\text { envíos }\end{array}$ \\
\hline Antonio Sánchez & 93.75 & $\begin{array}{l}\text { No hay } \\
\text { envíos }\end{array}$ & 100 & 76 \\
\hline Juan Zevallos & 92.11 & 90 & 100 & 100 \\
\hline
\end{tabular}

Nota: Sobre total de envíos anuales de cada uno.

Fuente: elaboración propia con base en Registro de Guías y Tornaguías de Asunción, en ANA, Nueva Encuadernación.

\section{CUADRO 3. GRANDES PROPIETARIOS DE BARCOS. TRANSPORTE ANUAL DE YERBA (EN ARROBAS)}

\begin{tabular}{lrrrrr}
\hline Dueño & 1783 & 1785 & 1786 & 1787 & 1789 \\
& & & & & \\
Luis Rodrigo Baldovinos & 0 & 17002.0 & 1402.0 & 8706.0 & 15578.0 \\
José González de los Ríos & 13926.5 & 0 & 14042.0 & 5663.5 & 11404.0 \\
Juan Machain & 31807.0 & 5618.0 & 0 & 15682.0 & 7777.5 \\
Tomás Ortega Fernández & 0 & 10140.0 & 9557.0 & 8195.0 & 0 \\
Antonio Sánchez & 8203.5 & 0 & 0 & 1929.0 & 8965.5 \\
Juan Zevallos & 23794.5 & 6374.0 & 22498.5 & 12461.5 & 17388.5 \\
\hline
\end{tabular}

Fuente: elaboración propia con base en Registro de Guías y Tornaguías de Asunción, en ANA, Nueva Encuadernación.

Varias son las razones que pueden explicar esa disparidad. Una a tener en cuenta es la participación simultánea de algunos de ellos como asentistas o particulares al servicio de la Real Renta de Tabacos. En 1780, por ejemplo, se aceptó la sociedad formada por Luis R. Baldovinos, Juan Machain y Juan Zevallos para proceder en conjunto o individualmente como asentistas, ello implicaba el compromiso de utilizar dos de sus bar- 
cos grandes para llevar tabaco río abajo entre los meses de octubre y marzo. ${ }^{21}$ Entre 1787 y 1794 el producto se remitió en barcos y lanchas particulares. Si se tiene en cuenta que el flete por la arroba de tabaco era de dos reales y el de yerba era de un real y medio, es comprensible considerar que, abocados a esa tarea más redituable, no podían a la vez disponer de transporte para la yerba. Otra razón son los totales registrados que no siempre resultan exactos, ya que los pilotos solían inscribir las cargas como si la embarcación fuese suya, por lo que no le es consignada al verdadero dueño. La salvedad, por lo menos en forma parcial, es para el caso de Juan de Zevallos, de quien se ha podido identificar a dos de dichos pilotos: Juan Francisco Centurión y Juan José Vera. Así como se ha mencionado el caso de santafesinos que viajaban con sus embarcaciones a Asunción a llevar productos y retornar con yerba y otras mercaderías paraguayas, se debe hacer referencia a transportadores que se trasladaban desde Buenos Aires llevando efectos de Castilla y que volvían con los frutos locales; como ejemplo se encuentra Antonio Melián, quien en 1786 llevó en su lancha sal y miel hacia Corrientes, yerba, dulce, miel y almidón a Santa Fe y dulce, miel, sal, azúcar y yerba a Buenos Aires; en total cargó 168 arrobas de dulce, 155 arrobas de azúcar, 22 arrobas de sal, 257 arrobas de miel y 10839 arrobas de yerba, ${ }^{22}$ y un año después cargó con destino a Buenos Aires 12910 arrobas de yerba, 24 suelas curtidas, 98 cajoncitos de conserva y tres arrobas de almidón. Si se tiene en cuenta que sólo en 1783 Cuello cargó para Santa Fe 2926 arrobas de yerba y Candioti 4132 arrobas, ${ }^{23}$ mientras que Tarragona en 1785 llevó lo ya apuntado (7 603 arrobas) y Melián lo indicado en este párrafo, se puede inferir que esas, como las acciones de otros transportistas no paraguayos, afectarían las cargas de los locales. Otra de las razones a tener en cuenta para explicar la disparidad en los embarques es la diversidad de actividades que estos transportistas realizaban y que les insumían tiempo y dedicación.

El panorama resulta más claro si se realiza una reseña para cada uno de ellos. Luis Rodrigo Baldovinos, considerado uno de los mayores transportistas, había obtenido una merced de tierras para estancia en el camino hacia el sur, en la zona de Saladillo $;^{24}$ también poseía una chacra entre Villeta y Asunción y residía en la ciudad capital. Tuvo una extensa familia, y parte de sus componentes (Rafael, Joaquín) participaban de sus negocios y también fueron funcionarios. Fue alcalde ordinario y síndico de la Santa Recolección. Al igual que otros transportistas, unió intereses mercantiles

${ }^{21}$ Caballero, Moneda, 2006, pp. 265 y 267.

${ }^{22}$ ANA, Nueva Encuadernación, vol. 1.

${ }^{23}$ Ibid., vol. 11.

${ }^{24}$ En la zona de la actual Villa Oliva. 
de intercambio con la propiedad de embarcaciones y tuvo recursos como para ser fiador de otros comerciantes. Tanto en barcos propios como ajenos, comerció con Buenos Aires y Santa Fe. Entre 1785 y 1788 cargó en total, a su consignación, 1057.5 arrobas, y a cargo de otros 2134 arrobas de yerba y 4315 varas de tirantes, dos de estos registros en barcos ajenos, uno de los cuales pertenecía a su yerno Pablo Urbieta. Resulta curioso que en tres de los registros de 1785 se identificó como alcalde ordinario, puesto que son los únicos, de todos los años compulsados, en los que hace alusión a su cargo capitular.

Otro transportista analizado es José González de los Ríos, originario de Andalucía, que vivía en el centro de Asunción, en las inmediaciones de la catedral. Entre otras funciones capitulares ocupó el cargo de alcalde de primer voto y poseía una estancia en la jurisdicción de Pilar de Ñeembucú. No sólo se dedicó al transporte de mercaderías ajenas en sus embarcaciones de gran porte, sino que también las empleó para sus propios negocios. En efecto, en los años 1783, 1786, 1787 y 1789 se encuentra que envía de su cuenta, en sus barcos, yerba, azúcar, algodón, miel y maderas, la mayoría a entregar en Buenos Aires a su pariente José Alberto Echeverría. También utilizó la garandumba de Baldovinos y la itapá de Santiago Báez para que le transportaran miel y yerba a Buenos Aires, en este último caso su fiador fue Ortega Fernández. No deja de ser curioso que en 1783 este realizó su última carga en el mes de junio, posible fecha de partida, y en el mes de julio don Pedro Echeverría declara que doña Antonia Echeverría le manda a su esposo, que era González de los Ríos, 30 arrobas de yerba en el barco de Antonio Sánchez. ${ }^{25} \mathrm{Al}$ igual que otros transportistas intervino como fiador de otras personas. Tal vez no sea de los más representativos entre los casos que se presentan, pero no es despreciable su participación y sus vinculaciones familiares y comerciales en Asunción y Buenos Aires, especialmente a través de su matrimonio.

En lo que se refiere a Juan Machain y Latiegui, vizcaíno, supo relacionarse con la más alta aristocracia asuncena por medio de su esposa doña Josefa Petrona Cálcena Echeverría. En 1780 ya era integrante del Cabildo, donde ocupó cargo a lo largo de varios años, y para 1783 era síndico de los Santos Lugares. ${ }^{26}$ En 1790 se pidió que fuera confirmado como capitán del puerto de Asunción, solicitud que le fue denegada al año siguiente. Era cosechador de tabaco, comerciaba con yerba y madera en sus propios barcos y en los de otros transportistas; como Ortega Fernández y Baldovinos ${ }^{27}$ fue frecuente que apareciera como fiador. Su flota de barcos, integrada según la

\footnotetext{
${ }^{25}$ ANA, Nueva Encuadernación, vol. 11, registro 205.

${ }^{26}$ Ibid., vol. 11, registro sin número del 12 de marzo.

${ }^{27}$ Ibid., vols. 11, 150 y 211.
} 
época por piragua, barco y garandumba, se amplió cuando en 1798 , siendo alcalde de primer voto, encargó la construcción de un bergantín "luego de haber calculado el lucro que ha de producirle al auxilio de las muchas proporciones del país que no se encontrarán en otro alguno". ${ }^{28}$

En consonancia con los cambios poblacionales surgidos a partir de la expansión hacia el norte con la fundación de la Villa de la Concepción y su rápido crecimiento, Machain diversificó sus rutas; en 1788 realizó un viaje a Buenos Aires, pero también envió embarcaciones a la villa, llevando cueros, vituallas, lienzo, algodón y efectos de Castilla enviados por Zevallos. Para 1801 su hijo Juan José trajinaba con una lancha, mientras Juan aparecía cargando su bergantín. ${ }^{29}$ Un año después el bergantín sigue a su nombre, pero la rendición de carga la firma su hijo por pedido de su madre, y denuncia un envío de 750 arrobas de yerba de la testamentaria de su padre. De tal manera que todo lo registrado a partir de esa fecha corresponde a la familia y herederos de Juan de Machain y Latiegui, luego de su muerte.

A su vez, Tomás Ortega Fernández, español, llegó a Paraguay como militar, tarea que nunca abandonó, como lo demuestra el haber sido comandante del fuerte San Carlos de Apa en 1801. A la vez estaba en el tema ganadero, ya que en 1789 envió una balsa a Corrientes con una carga de yerba para don Pedro Pérez, a quien también le envió doce armajes de taburetes de regalo para la casa y una carretilla que debía volver con la gente que mandaba a conducir ganado,${ }^{30}$ mientras que un año antes había remitido a Concepción 31 cueros y 40 arrobas de algodón en el barco de Antonio Sánchez. ${ }^{31}$ También comerciaba con Buenos Aires en sus propias naves o embarcándolas en las de otros; en 1783, por ejemplo, no realizó viajes con sus embarcaciones, pero no por ello dejó de comerciar, ya que envió de su cuenta dos cargas a Buenos Aires, una en barcos de Zevallos y otra en los de Machain; de la misma manera lo hizo en 1789, cuando envió en el bote de Luis Baldovinos quince tercios de yerba (112.5 arrobas) a la consignación de don Manuel Basabe, de quien era único abastecedor. ${ }^{32}$

En la década de 1790 Ortega ejerció varios cargos y tareas encomendadas por la autoridad; en su calidad de capitán fue nombrado juez de policía cuando el gobernador Lázaro de Ribera creó el cargo; ${ }^{33}$ en 1798, ocupando

${ }^{28}$ ANA, Historia, vol. 148, núm. 8, f. 12.

29 ANA, Nueva Encuadernación, vol. 1770.

${ }^{30}$ Ibid., vol. 211, registro 151.

${ }^{31}$ Ibid., vol. 11, registros 66 y 67, y vol. 150, y registro 416.

${ }^{32}$ Ibid., vol. 211, registro 219. Manuel Basabe se dedicaba al comercio y por herencia de su esposa poseía tierras en la campaña norte bonaerense, las mismas no estuvieron destinadas a la explotación agropecuaria sino a la especulación inmobiliaria

${ }^{33}$ Caballero, Bandos, 2007, p. 26. 
la función de alcalde de segundo voto, fue el encargado de tomar declaraciones para determinar si era conveniente el establecimiento de astilleros ${ }^{34}$ en 1809 , teniendo en cuenta "la inteligencia, actividad y celos por el R[ea]l. Servicio", fue designado director de la Fábrica de Cables al morir su titular Antonio Sánchez, de quien había sido ayudante, ${ }^{35}$ y en febrero de 1810 pasó a encargarse, por orden del gobernador intendente, de un juzgado de vigilancia para perseguir a los posibles simpatizantes de los franceses. ${ }^{36}$ A pesar de tanta actividad, el comercio y transporte de mercancías siguió siendo importante en su desempeño. En el rubro mercantil se encuentran envíos de su cuenta en sus embarcaciones y en las de otros con destino a Corrientes, consignados a Pedro Pérez, de quien también es fiador en ocasiones, y a Buenos Aires a la consignación del ya mencionado Manuel Basabe; en el primer caso manda efectos de Castilla y en el segundo yerba. ${ }^{37}$ También continuó el transporte en sus embarcaciones; para 1802, por ejemplo, en su bergantín Nuestra Señora del Rosario y San Jerónimo cargó 12224 arrobas de yerba. ${ }^{38} \mathrm{Al}$ morir poseía una estancia y una casa. ${ }^{39}$

En lo que corresponde a Antonio Sánchez, originario de Murcia, se puede decir que fue el más polifuncional de todos los analizados. No sólo se dedicó al transporte de mercadería en barcos propios que él mismo capitaneaba, sino que también era experto carpintero de barcos, armador y constructor de navíos, fue comerciante, realizó explotación forestal, capitán de milicia y, en 1797, fue designado director de la Fábrica de Cables y Calabrotes, cargo que ocupó hasta su muerte en 1809.

En 1801 Sánchez elevó un petitorio solicitando cobrar un sueldo permanente, pues el cargo de director sólo se le pagaba por los días que la fábrica funcionaba, según el requerimiento que hubiese de amarras y calabrotes; según él ese cargo lo alejaba de su trabajo de capitán de río y le ocasionaba serias pérdidas ya que: "Desembarazado al cabo de año y medio de esta ocupación [fabricar cables] hize un viaje en mi Barco, que lo tube parado todo esse tiempo, perdiendo el lucro y ganancias qe me redituan los fletes: no bien regresé a esta Provincia [se encargaron nuevos cables] postergando la atención de mi Barco que estaba dispuesto a seguir viaje." 40

El expediente que se inició aporta datos de interés respecto a la actividad del transportista, pues poseía un barco que capitaneaba personal-

\footnotetext{
${ }^{34}$ ANA, Historia, vol. 148, núm. 8.

${ }^{35}$ Ibid., vol. 210, núm. 11.

${ }^{36}$ Caballero, Bandos, 2007, p. 42.

${ }^{37}$ ANA, Nueva Encuadernación, vols. 11, 79, 1, 65, 150 y 211.

${ }^{38}$ Ibid., vol. 545.

${ }^{39}$ Durán, "Inmigrantes", 2011, p. 205.

${ }^{40}$ ANA, Nueva Encuadernación, vol. 1770.
} 
mente, ya que así ahorraba el sueldo de un sobrecargo, la comisión a un apoderado en Buenos Aires y otros gastos, pues los negocios los manejaba por sí mismo. Si se accedía a su pedido, podría asentarse de manera permanente en Asunción, evitando las demoras que se producían cuando el pedido de cables se llevaba a cabo cuando él estaba de viaje. A la vez se comprometía a atender cualquier otro requerimiento que se le hiciera en los temas de navegación.

El pedido se resolvió favorablemente recién en 1805, no obstante, no puede suponerse que su numerosa familia tuviese problemas de subsistencia, como él alegaba, si se tiene en cuenta que ese mismo año había invertido más de 3000 pesos en la Real Compañía Filipina en Buenos Aires, dedicada al tráfico esclavista. ${ }^{41}$

En lo que hace a su trabajo de transportista, no sólo llevó cargas ajenas, de las que en ocasiones fue fiador, sino que también condujo cargas a su propia consignación, como las 600 arrobas de yerba que, como vecino asunceno, envió en 1783, y las 1350 arrobas más 2000 pesos de plata por lo que se obligó en 1789. En 1785 no figuran registros en sus embarcaciones, pero no por ello dejó de comerciar; la garandumba de Miguel Caldevilla transportó a Buenos Aires, de cuenta y riesgo de Sánchez, 1098 arrobas de yerba, 38 tablas de cedro y ocho tirantes; a su vez en la balsa del padre presentado fray Francisco Xavier Castillo mandó a Santa Fe 343 arrobas para Juan Bautista del Castillo. En 1788 no realizó viaje a las provincias de Abajo, sino que lo hizo en dirección de Villa de la Concepción, llevando cueros, sebo, tabaco, sal, aguardiente, algodón, lienzo y herramientas. ${ }^{42}$

Respecto a Juan Zevallos, ${ }^{43}$ sus actividades más importantes se vincularon con el comercio y el transporte, aunque para 1779 entregó 500 arrobas de tabaco a la flamante Renta Real, de lo que se puede inferir que este producto se cultivaba en sus tierras o que era acopiador y revendía esta mercancía. Ocupó varias veces cargos capitulares, incluido el de alcalde de primer voto, entre 1807 y 1808 fue diputado provincial del Consulado de Buenos Aires y, producidos los acontecimientos de 1811, formó parte del primer gobierno revolucionario; también fue subdelegado de bienes difuntos y apoderado del pueblo de Santa Rosa. Su sólida posición económica se puede deducir del hecho de que, al morir, poseía dos casas, una estancia, una chacra, ganado y nueve esclavos, más diversos bienes valuados, parcialmente, en algo más de 9000 pesos. $^{44}$

${ }^{41}$ Cooney, Economía, 1990, p. 81.

${ }^{42}$ ANA, Nueva Encuadernación, vols. 11, 211, 79 y 150.

${ }^{43}$ El segundo nombre, Valeriano, lo utilizaba esporádicamente.

${ }^{44}$ Durán, "Inmigrantes", 2011, p. 204. 
En su condición de transportista fue uno de los más importantes por cantidad de viajes y carga acumulada, de la que en varias ocasiones fue fiador. En este caso los cálculos son más confiables pues, como ya se ha dicho, a lo largo de los años se puede identificar en los registros a dos de sus pilotos, Juan Francisco Centurión, su sobrino, y Juan José Vera, ${ }^{45}$ quienes generalmente figuraban en calidad de transportistas, sin aclarar que lo hacían en barcos de Zevallos. Fue frecuente que hiciera envíos por su cuenta de yerba, azúcar, maderas y, en menor cantidad, algodón y aguardiente. Las cargas las hacía tanto en sus embarcaciones como en las de otros, los destinos eran Buenos Aires y Santa Fe, en este último caso a personas con quienes estaba emparentado, como Candioti. En 1786 mandó de su cuenta a dos consignatarios un total de 4805 arrobas de yerba, y al año siguiente, en embarcaciones de diferentes dueños, 3124 arrobas de la misma mercancía. Si bien no se han encontrado registros que indiquen que Zevallos hacía viajes al norte, sí se sabe que los efectos de Castilla que llevaba desde Buenos Aires podían tener como destino la Villa de la Concepción. ${ }^{46}$

Como se ha mencionado en cada reseña y según el cuadro 4, puede apreciarse que todos los transportistas analizados poseían puntos en común que les permitían ejercer poder y control de los intercambios. En concordancia con lo afirmado por varios autores ${ }^{47}$ la última etapa del dominio hispano en Asunción se caracterizó por el afianzamiento de recién llegados en detrimento del control ejercido hasta entonces por la población tradicional, que continuó la actividad rural y comercial pero se vio muy restringida en el manejo de la carrera de Paraguay, de la que participó a través de sus contactos mercantiles, ya que integrantes de familias como los Recalde, los Flecha, los Vigil, los de la Mora, los Esquivel o los Bogarín, por mencionar sólo algunas, siguieron desempeñándose como fiadores y comerciando, a veces utilizando sus propias lanchas, garandumbas o piraguas, pero no pudieron hacer frente al poderío económico de los recientemente establecidos, aunque sí conservaron la exclusividad de sus clientes. Por ejemplificar con algunos casos, basta mencionar que Domingo Belgrano se abastecía por medio de Antonio María Esquivel; Manuel Basavilbaso lo hacía especialmente a través de Pedro José Recalde; José Riera mantuvo la conexión con Agustín Trigo, y Cristóbal Aguirre recibía su cuantiosa provisión de

\footnotetext{
${ }^{45}$ En el registro 210 del 16 de septiembre de 1786 se hace la aclaración de que se carga en el barco de Zevallos que lleva su piloto Centurión. Del mismo modo, cuando en 1791 se declara la carga del barco San Agustín de Juan Valeriano Zevallos, se indica que el piloto es Vera, en ANA, Nueva Encuadernación, vols. 1 y 545, f. 133, y en Testamento de Juan Baleriano Zevallos y Tomasa Rodríguez de Vega (1812), en ANA, Libro de Testamentos, vol. 702, núm. 2.

${ }^{46}$ En 1788, por ejemplo, Juan Machain llevó en su barco a Concepción los efectos de Castilla que enviaba Zevallos. Véase ANA, Nueva Encuadernación, vol. 150, registro 236.

${ }^{47}$ Por ejemplo, Cooney, Economía, 1990, y Areces y Bouvet, "Libertad", 2001.
} 


\section{CUADRO 4. COINCIDENCIAS DE LOS TRANSPORTISTAS ANALIZADOS}

\begin{tabular}{lcccccc}
\hline Transportista & Transporte & Comercio & Fiador & $\begin{array}{c}\text { Cabildo } y / 0 \\
\text { funcionario }\end{array}$ & Tierras & $\begin{array}{c}\text { Carrera } \\
\text { militar }\end{array}$ \\
$\begin{array}{c}\text { Luis Rodrigo } \\
\text { Baldovinos }\end{array}$ & $\mathrm{X}$ & $\mathrm{X}$ & $\mathrm{X}$ & $\mathrm{X}$ & $\mathrm{X}$ & \\
$\begin{array}{l}\text { José González } \\
\text { de los Ríos }\end{array}$ & $\mathrm{X}$ & $\mathrm{X}$ & $\mathrm{X}$ & $\mathrm{X}$ & $\mathrm{X}$ & \\
Juan Machain & $\mathrm{X}$ & $\mathrm{X}$ & $\mathrm{X}$ & $\mathrm{X}$ & $\mathrm{X}$ & $\mathrm{X}$ \\
$\begin{array}{c}\text { Tomás Ortega } \\
\text { Fernández }\end{array}$ & $\mathrm{X}$ & $\mathrm{X}$ & $\mathrm{X}$ & $\mathrm{X}$ & $\mathrm{X}$ & $\mathrm{X}$ \\
$\begin{array}{c}\text { Antonio } \\
\text { Sánchez }\end{array}$ & $\mathrm{X}$ & $\mathrm{X}$ & $\mathrm{X}$ & $\mathrm{X}$ & $\mathrm{X}$ & $\mathrm{X}$ \\
Juan Zevallos & $\mathrm{X}$ & $\mathrm{X}$ & $\mathrm{X}$ & $\mathrm{X}$ & $\mathrm{X}$ & $\mathrm{X}$ \\
\hline
\end{tabular}

Fuente: elaborado con base en la documentación mencionada entre las notas 25 y 45.

maderas por medio de Santiago Báez y la yerba especialmente por Antonio Vigil, Florencio Zelada y Manuel García de Arze. ${ }^{48}$

Otra de las observaciones de los autores mencionados en el párrafo anterior es la dependencia que los comerciantes de Paraguay tenían respecto a los de Buenos Aires. Si bien las pruebas documentales así lo confirman, se considera que, en algunos casos, lo que se puso en práctica fue una estrategia de tipo familiar, con representantes en Paraguay y en Buenos Aires, en que el origen era asunceno y no porteño, como fue el caso de la familia Cálcena Echeverría (véase cuadro 5).

El matrimonio paraguayo Cálcena Echeverría y Aguiar, de las familias tradicionales del lugar, casaron a sus hijos con integrantes de preeminencia; dos de las mujeres contrajeron matrimonio con transportistas y comerciantes españoles firmemente establecidos en Asunción, y sus hijos varones con descendientes de estirpe del más alto nivel, como los Recalde y los De la Mora.

José Alberto, si bien viajaba frecuentemente a Paraguay, se estableció de manera permanente en Buenos Aires a partir de 1783, dos años después también se trasladaron a esta ciudad su esposa e hijos. En la capital virreinal recibía los envíos de sus cuñados Juan Machain y José González

\footnotetext{
${ }^{48}$ Los registros de los que se extraen estas conclusiones se encuentran en ANA, Nueva Encuadernación, en los volúmenes correspondientes a cada año.
} 


\section{CUADRO 5. RELACIONES DE LA FAMILIA CÁLCENA ECHEVERRÍA Y AGUIAR}

\begin{tabular}{|c|c|c|}
\hline $\begin{array}{l}\text { Juan José Cálcena Echeverría } \\
\text { (España, 1729-Asunción, 1806) }\end{array}$ & casado con & $\begin{array}{c}\text { Clara Aguiar y Aberstain } \\
\text { (Santa Fe, 1736-Asunción, 1805) }\end{array}$ \\
\hline $\begin{array}{l}\text { José Alberto } \\
\text { (Santa Fe, 1751) }\end{array}$ & $\begin{array}{l}\text { Hijos } \\
\text { casado con }\end{array}$ & $\begin{array}{c}\text { María Agueda Recalde } \\
\text { (Paraguay) }\end{array}$ \\
\hline $\begin{array}{l}\text { María Antonia } \\
\text { (Santa Fe, 1752) }\end{array}$ & casada con & $\begin{array}{l}\text { José González de los Ríos } \\
\text { (España) }\end{array}$ \\
\hline $\begin{array}{l}\text { Josefa Petrona } \\
(\text { Santa Fe, 1755) }\end{array}$ & casada con & $\begin{array}{l}\text { Juan Machain Latiegui } \\
\text { (España) }\end{array}$ \\
\hline Pedro José & casado con & Juana Josefa de la Mora y \\
\hline (Paraguay, 1757) & & Casal (Paraguay) \\
\hline $\begin{array}{l}\text { Rosa Buenaventura } \\
\text { (Paraguay, 1758) }\end{array}$ & casada con & $\begin{array}{l}\text { José Antonio de Astigarraga } \\
\text { (Paraguay) }\end{array}$ \\
\hline
\end{tabular}

Fuentes: "Juan José Cálcena y Echeverría" y "Clara Aguiar y Aberstain" en <www.geni. com $>\mathrm{y}<\mathrm{www}$.genealogiafamiliar.net $>$. [Consulta: abril de 2013.]

de los Ríos, de su hermano Pedro José y de otros proveedores, algunos de los cuales también eran parientes, aunque no tan cercanos (véase cuadro 6). Es válido suponer que en realidad no eran las transacciones entre un comerciante dominante de Buenos Aires y un transportador y comerciante paraguayo, sino que era un único negocio megafamiliar.

Otros comerciantes mantenían relaciones diferentes. En el caso de Zevallos, ya se indicó su relación con Candioti en Santa Fe; en Buenos Aires, aunque no en forma excluyente, afianzó sus vinculaciones con Miguel González Noriega, Pedro González Cortina y Román Ramón Díaz, todos cuñados entre sí y comerciantes importantes de Buenos Aires, así como también con Francisco Díaz Parafán (véase cuadro 7).

En el caso de Baldovinos se repite la vinculación con González Noriega y con la sociedad formada por Pedro González Cortinas y Juan Llanos, y también se relaciona con José San Pedro Llorente, comerciante mayorista de la capital, aunque es de destacar que el contacto comercial con estos representantes del comercio porteño es mayoritariamente de maderas. $\mathrm{Si}$ bien en un embarque de 1787 hay 2452 arrobas de yerba consignadas a González Noriega, en ese mismo año también le envía, en dos cargas, un total de 1770 varas de tirantes; un año antes se registra un solo embarque de Baldovinos a su nombre, y es de 1400 varas de tirantes para el mismo González Noriega. 
CUADRO 6. CARGAS DE YERBA (EN ARROBAS) RECIBIDAS POR JOSÉ ALBERTO ECHEVERRÍA EN BUENOS AIRES

\begin{tabular}{|c|c|c|c|c|c|}
\hline$A \tilde{n} o$ & $\begin{array}{c}\text { Total } \\
\text { recibido }\end{array}$ & $\begin{array}{c}\text { Juan } \\
\text { Machain } \\
\text { (cuñado) }\end{array}$ & $\begin{array}{c}\text { José González } \\
\text { de los Ríos } \\
\text { (cuñado) }\end{array}$ & $\begin{array}{l}\text { Pedro José } \\
\text { (hermano) }\end{array}$ & Otros \\
\hline 1783 & 16440.0 & 16440.0 & No envía & No envía & No envían \\
\hline 1785 & 1722.0 & 1722.0 & No envía & No envía & No envían \\
\hline 1786 & 120.0 & No envía & No envía & No envía & 120.0 \\
\hline 1787 & 9670.5 & 8385.0 & 783.0 & No envía & 502.5 \\
\hline 1788 & 8927.0 & 4522.0 & No envía & 4132.5 & 272.5 \\
\hline 1789 & 19252.0 & 8542.5 & 2880.0 & No envía & 7830.0 \\
\hline \multicolumn{6}{|c|}{ Total seis } \\
\hline años & 56311.5 & 39611.5 & 3663.0 & 4132.5 & 8725 \\
\hline
\end{tabular}

Fuente: elaboración propia con base en Registro de Guías y Tornaguías de Asunción, en ANA, Nueva Encuadernación.

CUADRO 7. VINCULACIÓN ZEVALLOS-GONZÁLEZ NORIEGA Y VINCULACIÓN ZEVALLOS- DÍAZ PARAFÁN (EN ARROBAS)

\begin{tabular}{lcccc}
\hline Envíos de yerba & A González & & \\
Año & de su cuenta & Noriega & A Díaz Parafán & A otros \\
1785 & 3287 & 0 & 2377 & 910 \\
1786 & 4805 & 4108 & 0 & 697 \\
1787 & 3124 & 1404 & 975 & 745 \\
\hline
\end{tabular}

Fuente: elaboración propia con base en Registro de Guías y Tornaguías de Asunción, en ANA, Nueva Encuadernación.

Respecto a Ortega Fernández, ya se ha indicado que sus contactos estaban en Buenos Aires por medio de Manuel Basabe. Su otro punto de intercambio estaba en Corrientes, por medio de Pedro Pérez, a quien no sólo abastecía de efectos de Castilla, sino que también le enviaba regalos de productos locales y le salía de fiador.

A medida que avanzaba la década de 1790 se puede apreciar que estos transportistas comienzan a mermar en los registros; aunque mantuvieron embarques hasta los primeros años del siglo XIX, empezaron a aparecer 
nuevos dueños que los fueron reemplazando. En el caso de Machain se ha visto cómo su hijo continúa con la actividad luego de la muerte de su padre en 1802, algo similar ocurre con Baldovinos, y en el caso de Zevallos aumentan las cargas de Juan Francisco Centurión, de lo que se podría inferir que, atento al tipo de parentesco que los unía, llegó a asociarse con su antiguo empleador. ${ }^{49}$ A su vez surgen nombres nuevos, que sugieren la posibilidad de una renovación, lo que puede dar pie a la continuación de esta línea de investigación.

\section{ConClusiones}

A los cambios vividos por Paraguay en el último tercio del siglo XVIII se agregó, en no menor importancia, la participación de un grupo de "extraños", al punto que casi monopolizó el transporte en la carrera de Paraguay. Esos nuevos "dueños del transporte" no se limitaron a ser simples cobradores de fletes, sino que fueron comerciantes ellos mismos, sus recursos les permitían ser fiadores de las personas con las que mantenían más estrechas relaciones en Paraguay, a la vez que se convirtieron en funcionarios y participantes del Cabildo en su más alta jerarquía.

Los lazos familiares de los transportistas recientemente llegados les permitieron expandir sus negocios y hacer pie en la elite comercial tanto de Buenos Aires como de Santa Fe, al establecer uniones matrimoniales con familias tradicionales que, sin abandonar su lugar de preeminencia en Asunción, se insertaron en la capital virreinal, manejando sus propios negocios. Los grandes transportistas se relacionaban entre ellos, tanto en el aspecto comercial como social, ya que formaban sociedades, se desempeñaban como fiadores intragrupo, establecían lazos familiares y compartían sus actividades.

Las familias tradicionales paraguayas dejaron de dominar el transporte pero mantuvieron sus lazos económicos con Buenos Aires, al que proveían no sólo de yerba, sino también de maderas y, en menor medida, de azúcar, utilizando en ocasiones sus propias embarcaciones, pero cargando cada vez más frecuentemente en los barcos de los grandes transportadores.

La expansión poblacional paraguaya, con el establecimiento de nuevas villas como la de Concepción y la de Ñeembucú, abrió nuevos mercados tanto para los productos de Castilla que recibían desde Buenos Aires,

\footnotetext{
${ }^{49}$ Juan Francisco Centurión no sólo era uno de los sobrinos de Zevallos (quien no tenía hijos biológicos), sino que se casó con la hija adoptiva de este, Antonia, quien años después fue declarada única y universal heredera del matrimonio formado por Juan Baleriano Zevallos y Tomasa Rodríguez de Vega. ANA, Libro de Testamentos, vol. 702, núm. 2.
} 
como para los productos locales, con los que era necesario abastecer estos centros recién creados. A medida que finalizaba el siglo fue notorio que se produjo una renovación y que estos grandes transportistas fueron siendo reemplazados por otros más jóvenes, en ocasiones sus herederos, y en otras oportunidades por nuevos interesados en mantener el comercio con las provincias de Abajo.

Todo lo expuesto pone de manifiesto un Paraguay dinámico, alejado de la visión habitual de aislamiento y estancamiento. El dinamismo provino tanto de la población de honda raigambre como de los migrantes de fines del siglo XVIII, lo que deja en claro que la rivalidad entre ambos sectores de la sociedad no fue tan profunda como usualmente se ha dicho. De la misma manera se puede afirmar que la dependencia de Asunción respecto de Buenos Aires existió, pero en menor proporción al supuesto que en tal sentido se impuso en la historiografía tradicional.

Algunos de los factores que contribuyeron para tales logros se pueden encontrar en la existencia de productos exclusivos, como la yerba mate, el tabaco y las maderas de alta calidad; en la llegada de hombres con recursos propios que inyectaron metálico y conocimientos para el emprendimiento de nuevas actividades; en la habilidad para tejer redes familiares que permitieran unir la posesión de tierras con el prestigio social y las vinculaciones mercantiles.

Posicionarse en Asunción y desde allí proyectar la visión hacia el sur permite analizar la realidad paraguaya de la época intendencial con criterios distintos al porteñocentrismo, ampliando así la perspectiva regional como un conjunto de subregiones articuladas entre sí que funcionaban con interdependencia, a la vez que formaban parte de un entramado mercantil intra e interregional que permitía la conexión virreinal y ultramarina para ubicar los productos paraguayos.

Respecto al objetivo de contribución historiográfica propuesto en el comienzo de este trabajo, cabe aclarar que el presente es un paso más en la búsqueda de integrar las temáticas de Paraguay de fines del siglo XVIII. Sin embargo, por ser este un proyecto que excede los límites de un artículo, se buscará que, en un futuro cercano, se pueda armar una exposición totalizadora con todas las investigaciones realizadas por la autora en los últimos cinco años, para darlas a conocer en conjunto.

\section{FUENTES CONSULTADAS}

\section{Archivos}




\section{Bibliografía}

Aguirre, Juan Francisco, Discurso histórico sobre el Paraguay, 1793, estudio preliminar y restitución del texto por Ernesto J. A. Maeder, Buenos Aires, Academia Nacional de la Historia, 2003.

Alvear, Diego De, Relación histórica y geográfica de la Provincia de Misiones (1785), Resistencia, Instituto de Investigaciones Geohistóricas/Consejo Nacional de Investigaciones Científicas y Técnicas, 2000.

Areces, Nidia R., Estado y frontera en el Paraguay. Concepción durante el gobierno del Dr. Francia, Asunción, Universidad Católica Nuestra Señora de la Asunción, 2007.

y Nora E. Bouvet, "La 'Libertad de los tabacos' y las relaciones entre Asunción y Buenos Aires, 1810-1813”, Boletín Americanista, Universitat de Barcelona, núm. 52, 2001, pp. 57-83.

Assadourian, Carlos Sempat, El sistema de la economía colonial. El mercado interior. Regiones y espacio económico, México, Nueva Imagen, 1983.

Atlas escolar universal, Buenos Aires, Granda, 1977.

AzARA, FÉLIX DE, Diario de la navegación y reconocimiento del río Tebicuarí (1785), Buenos Aires, Imprenta del Estado, 1836. En Biblioteca Antológica, Romanticismo, Hispanoamerica, Crónica, en <http://www.biblioteca-antologica.org/>. [Consulta: 20 de mayo de 2012.]

BARSKY, ANDRÉS, “Auge y ocaso de las 'Regiones geográficas argentinas' de Federico Daus. De un pasado con certezas a una actualidad de fragmentación" en Anuario de la División Geografía 2000-2001, Luján, Universidad Nacional de Luján, 2001, pp. 35-48.

Caballero Campos, Herib, "Cosecheros, contrabandistas y consumidores: el comercio del tabaco entre Asunción y Buenos Aires 1779-1811” en Liliana M. Brezzo (ed.), Aislamiento, nación e historia: Argentina y Paraguay. Siglos XVIII-XX, Rosario, Universidad Católica Argentina, 2005, pp. 75-133.

De moneda a mercancía del rey. Efecto y funcionamiento de la Real Renta de Tabaco y Naipes en la Provincia del Paraguay (1779-1811), Asunción, Arandura, 2006. Los Bandos de Buen Gobierno en la Provincia del Paraguay (1778-1811), Asunción, Arandura/Fondo Nacional de la Cultura y las Artes, 2007. , El proceso de la independencia del Paraguay 1780-1813, Asunción, El Lector, 2010.

Castañeda, Carlos E. y Jack Autrey Dabbs, Calendar of the Manuel E. Gondra Manuscript Collection, The University of Texas Library, México, Jus, 1952, en <http:// www.lib.utexas.edu/taro/utlac/00066/lac-00066p1.html>. [Consulta: 15 de abril de 2012.]

Cooney, Jerry W., Economía y sociedad en la Intendencia del Paraguay, Asunción, Centro Paraguayo de Estudios Sociológicos, 1990. 
"Los yerbatales del norte: la explotación de la frontera paraguaya, 1776-1810" en Whigham Thomas L. y Jerry W. Cooney, Campo y frontera: el Paraguay al fin de la era colonial, Asunción, Servilibro, 2006, pp. 35-61.

Djenderedjian, Julio C., "Economía y sociedad en la Arcadia criolla. Formación y desarrollo de una sociedad de frontera en Entre Ríos, 1750-1820”, tesis de doctorado, Universidad Nacional de Buenos Aires, 2003.

Durán, DiAnA, "Inmigrantes españoles en la Provincia del Paraguay. 1776-1811", Anuario del Instituto de Historia Argentina, Universidad Nacional de La Plata, núm. 11, 2011, pp. 183-206.

FERrer, Renée, Un siglo de expansión colonizadora. Núcleo poblacional establecido en torno a la Villa Real de la Concepción. Origen y desarrollo socio-económico, Asunción, Centro de Estudios Antropólogicos de la Universidad Católica, 2008.

Garavaglia, Juan C., Economía, sociedad y regiones, Buenos Aires, De la Flor, 1987. Mercado interno y economía colonial. Tres siglos de historia de la yerba mate. Rosario, Prohistoria, 2008.

LEvinson, Norberto, "Guaraníes y charrúas: una frontera exclusivista-inclusivista", Revista de Historia Regional, Universidade Estadual de Ponta Grossa, vol. 14, núm. 1, verano de 2009, pp. 49-75, en <http://www.revistas.uepg.br/>. [Consulta: 2 de junio de 2010.]

Maeder, Ernesto J. A., Historia económica de Corrientes en el periodo virreinal. 1776- 1810, Buenos Aires, Academia Nacional de la Historia, 1981.

y Ramón Gutierrez, Atlas histórico del nordeste argentino, Resistencia, Instituto de Investigaciones Geohistóricas, 1995.

Mariluz Urquijo, José M., El virreinato del Río de la Plata en la época del marqués de Avilés (1799-1801), Buenos Aires, Plus Ultra, 1987.

Musser, Karl, "Río Paraná", Wikimedia Commons, 17 de diciembre de 2009, en $<$ https://commons.wikimedia.org/wiki/File:Paranarivermap.png>. [Consulta: julio de 2013.]

PAREDES, ISABEL, "Las rutas mercantiles en el área rioplatense a mediados del siglo XVIII", XI Congreso SOLAR, Bahía Blanca, Sociedad Latinoamericana de Estudios sobre América Latina y el Caribe/Universidad Nacional del Sur, 2008.

"El comercio en la subregión de Paraguay a fines del siglo XVIII", XII Jornadas Interescuelas y/o Departamentos de Historia, San Carlos de Bariloche, 2009.

"La región rioplatense a fines del siglo XVIII. Interconexiones y relaciones poblacionales", XXX Encuentro de GeoHistoria Regional, [CD], Resistencia, Universidad Nacional del Nordeste, 2010, ISBN 978-987-26437-7-5.

, "Entre la ilegalidad y la necesidad: los contrabandistas en el Río de la Plata en la segunda mitad del siglo XVIII", XIIIJornadas Interescuelas/Departamentos de Historia, Catamarca, Universidad Nacional de Catamarca, 2011.

Quiroga, José, Descripción del río Paraguay, desde la boca de Xaurú hasta la confluencia del Paraná (1752), Buenos Aires, Imprenta del Estado, 1836 [en línea], en <www. cervantesvirtual.com>. 
REITANO, EMIR, "Veleros y garandumbas. La navegación del estuario rioplatense durante el periodo colonial tardío", Actas del VI Congreso Argentino de Americanistas, Buenos Aires, Sociedad Argentina de Americanistas, 2009, t. 1, pp. 445-470.

Rivarola PaOli, Juan B, La economía colonial, Asunción, Litocolor, 1986, en <http:// www.bvp.org.py/biblio_htm/rivarola/riv_2_iii_iv.htm>. [Consulta: 20 de agosto de 2012.]

La contabilidad colonial y las Cajas Reales de Hacienda, Asunción, Intercontinental, 2008.

Romero de Viola, Blanca Rosa, Paraguay siglo XVIII. Periodo de transición, Asunción, Comuneros, 1987.

Rosal, Miguel A., "El transporte hacia Buenos Aires a través de la hidrovía ParaguayParaná, 1781-1811”, Jahrbuch für Geschichte Lateinamerikas, Deutsche Forschungsgemeinschaft, núm. 27, 1990, pp. 127-147, en <www.gewi.uni_graz.at/jbla/>. [Consulta: 13 de abril de 2012.]

Rusiñol, Santiago, De Barcelona al Plata. Un viaje a la Argentina de 1910, Barcelona, Ediciones B, 1999.

SAguier, Eduardo R., Genealogía de la tragedia argentina, t. II, 2004/2007, en <http:// www.er-saguier.org/>. [Consulta: 31 de agosto de 2012.]

Telesca, Ignacio, "Tras los expulsos. Cambios demográficos y territoriales en el Paraguay después de la expulsión de los jesuitas”, tesis doctoral, Universidad Torcuato Di Tella, 2009.

Wentzel, Claudia, "El comercio del litoral de los ríos con Buenos Aires: el área del Paraná. 1783-1821”, Anuario IEHS, Universidad Nacional del Centro de la Provincia de Buenos Aires, núm. 14, 1988, Tandil, pp. 161-210.

Whigham, Thomas L., Lo que el río se llevó. Estado y comercio en Paraguay y Corrientes, 1776-1870, Asunción, Universidad Católica Nuestra Señora de Asunción, 2009.

y Jerry W. CoOney (eds.), Campo y frontera: el Paraguay al fin de la era colonial, Asunción, Servilibro, 2006. 